\title{
Characterization of GaSb thin films from tailor-made single source precursors
}

\author{
Andreas Seemayer*, Alexander Hommes, Sascha Hümann ${ }^{\dagger}$ and Klaus Wandelt \\ Institute for Physical Chemistry - University of Bonn \\ Wegelerstr. 12, 53115 Bonn, Germany \\ Stephan Schulz \\ Institute for Inorganic Chemistry - University of Essen \\ Universitätsstr. 6, 45117 Essen, Germany
}

\begin{abstract}
:
We investigated the growth and the surface properties of $\mathrm{GaSb}$ on $\mathrm{Si}(001)$ substrate by using a tailor-made fully-alkyl-substituted heterocyclic single-source precursor. The precursor properties during the evaporation process where monitored by RGA. The initial film growth was monitored by AES. Using a HV cold wall reactor, dense GaSb films could be produced and where characterized by AES, AFM and S-XPS. The results are discussed in terms of a correlation of the electronic and geometrical properties with the composition and structure of the films.
\end{abstract}

Note: This is a short version of the submitted abstract. The journal (Journal of Crystal Growth) does not accept formatted text in the abstract. The total length of the proceedings paper is limited to 4 pages. 


\section{Introduction}

III-V semiconductor films used for opto- and microelectronic devices have traditionally been grown by (MO)MBE and LPE processes. An alternative metal-organic CVD-Process, which has been established in the last two decades for high-throughput and low-cost fabrication works for nitrides, phosphides and arsenides, but is problematic for antimonides. In particular, for the production of $\mathrm{GaSb}$ thin films, an alternative synthesis route is a CVD-process using fully-alkyl-substituted heterocyclic single-source precursors of the general form $\left[\mathrm{R}_{2} \mathrm{GaSb} \mathrm{R}_{2}\right]_{\mathrm{n}}$.

Tetraalkyldistibines $\mathrm{Sb}_{2} \mathrm{R}_{4}$ tend to react with trialkylgallanes $\mathrm{GaR}_{3}$ under $\mathrm{Sb}-\mathrm{Sb}$ bond cleavage. The reaction proceeds stepwise with the initial formation of a distibine Lewis acid-base adduct, which subsequently reacts under $\mathrm{Sb}-\mathrm{Sb}$ and $\mathrm{Ga}-\mathrm{C}$ bond cleavage and formation of heterocyclic stibinogallanes. All presented experiments were performed by using the precursor $\left[{ }^{\mathrm{t}} \mathrm{Bu}_{2} \mathrm{GaSb} \mathrm{Et}_{2}\right]_{2}$ (1).

\section{Experimental}

The used precursor (1) were synthesized by a literature method [1] using standard Schlenck-techniques and a glovebox with $\mathrm{N}_{2}$ atmosphere as a storage. The compound was transferred into the UHV chamber by using a closed glass vessel.

In a first step, the precursor properties where monitored during the evaporation process under UHV conditions (base pressure $<10^{-9}$ mbar). Due to the very high cleanliness any influence of other species to the growth process could be excluded. The gas phase during the growth process was monitored via Residual Gas Analysis by using a quadrupole mass spectrometer (Balzers QMG 112) and the produced films were characterized using Auger Electron Spectroscopy (Perkin Elmer PHI 3017 Auger Subsystem). For sample cleaning 
the UHV chamber is equipped with a sputter gun, which produces an ion current of about $10 \mu \mathrm{A}$ on the sample, which is equivalent to a sputter rate of about $0,8 \mathrm{ML} / \mathrm{min}$.

Additional growth experiments were performed in a cold wall CVD reactor under HV conditions. The HV grown films were stored in glass vessels under ambient conditions and transferred to the just described UHV chamber.

Using a commercial PicoSPM I AFM in contact mode and in tapping mode, the morphology of the produced surfaces was investigated. All AFM measurements were made under ambient conditions.

The S-XPS measurements were performed at the SoLiAs UHV chamber at BESSY II. The sample were cleaned by sputtering with argon iones by using a beam energy of $500 \mathrm{eV}$ and a ionisation current of $20 \mathrm{~mA}$. All spectra were taken under normal emission. Under this condition the photoelectron escape depth is equal to the inelastic mean free path.

\section{Results and Discussion}

Due to former DSC results [2] the precursor molecule shows thermal decomposition at $464 \mathrm{~K}$ and $486 \mathrm{~K}$. Therefore it should be possible to sublime the species without decomposition at lower temperatures. Monitoring the base pressure during evaporation shows an increasing pressure at temperatures above $370 \mathrm{~K}$ (figure 1). By identifying the compounds fragments by residual gas analysis with the constraint of a reasonable growth rate but low background pressure of residual fragments which may lead to carbon contamination of the produced films, evaporation temperatures around $400 \mathrm{~K}$ were found to be a good process value.

In UHV, gallium and antimony could be deposited on $\mathrm{Si}(001)$ substrate, but due to the high contamination of the residual gas, an excess of antimony species was detected. During deposition the sample was heated to temperatures in the range of $670 \mathrm{~K}$ to $770 \mathrm{~K}$. In 
general lower deposition temperatures could be used, but then the quality of the produced films would be rather low in terms of full coverage [2]. After sputtering, the carbon contamination was eliminated (figure 2a), but the Sb:Ga ratio was still greater then unity. Therefore the carbon intoxication is a post deposition process, whilst the antimony excess leads to the assumption, that the precursor molecule undergoes some reactions in the gas phase. Comparing the adsorbent and substrate Auger intensities during sputtering a Frankvan-der-Merwe growth mode could be excluded (figure $2 b$ ).

Using a home-made cold wall MOCVD reactor, dense GaSb films of the thickness of about $1 \mu \mathrm{m}$ could be produced [2]. Comparison of the Auger spectra of the UHV- and HVgrown films shows that there is a chemical shift within the Sb MNN Auger electrons, which is due to the antimony excess during the UHV growth process (figure 3). The Auger intensities of the HV-grown films were constant within the grown area of about $1 \mathrm{~cm}^{2}$. Due to the transport the surface region was contaminated with carbon and oxygen species. Cleaning by argon ion sputtering leads to a carbon free surface after 40 minutes, whereas a sputter time of about 90 minutes was needed for cleaning the samples from any oxygen contamination.

The chemical composition of the HV-grown films was characterized by S-XPS. Due to the transport oxygen and carbon impurities were found to be present on top of the GaSb film. No other species could be identified on the surface. By using different primary energies, namely $150 \mathrm{eV}, 650 \mathrm{eV}$ and $1070 \mathrm{eV}$ the composition of the surface and the bulk region could be investigated due to the different escape depth of the produced photoelectrons. In particular by high resolution spectra the Sb4d, Sb3d and Ga3d photoelectrons were investigated. The impurities where characterized by $\mathrm{O} 1 \mathrm{~s}$ and $\mathrm{C} 1 \mathrm{~s}$ region scans. The presence of GaSb was proven due to the binding energy of the considered Ga and Sb peaks. They agree with reference spectra of pure GaSb (Sigma Aldrich 99,99\%) as well as literature data [3], [4]. The results are shown in table 1 and 
figure 4. The presence of pure $\mathrm{Ga}$ or $\mathrm{Sb}$ moieties could be excluded. The samples could be cleaned by argon ion sputtering within $20-40$ minutes. Due to the sputtering process the surface region was found to be gallium-rich in agreement with [5]. In the case of thin film production on pure $\mathrm{Si}(001)$ the bulk compound was found to be stöchiometric under consideration of experimental errors. When preoxidized silicon wafers are used, the films were found to be gallium-rich.

Due to geometry optimization in respect to former experiments [2], the surface roughness could be reduced by about 2 magnitudes. The topography is strongly dependent on the substrates temperature during the deposition process. The higher the temperature, the bigger the single crystallites are. At higher deposition temperatures the typical cubic shape of GaSb single crystals could be identified. At a deposition temperature of $723 \mathrm{~K}$ the average height is $58 \mathrm{~nm}$ and the RMS is $14.3 \mathrm{~nm}$. At a deposition temperature of $773 \mathrm{~K}$ the average height could be measured as $125 \mathrm{~nm}$ and the RMS value is $26 \mathrm{~nm}$. The roughness histograms of films which are grown at the presented substrate temperatures are shown in figure 5. If the growth process is initiated on preoxidized silicon wafers, an additional ordering effect takes place since all crystallites have the same spatial alignment. Measurements in non-contact mode did not show any contrast in the phase image. This means that there are besides GaSb no other gallium or antimony moieties present on the surface, which is in agreement with the AES and S-XPS results.

\section{Conclusion}

It has been shown that tailor-made single source precursors of the general type $\left[\mathrm{R}_{2}\right.$ $\left.\mathrm{GaSb} \mathrm{R}{ }_{2}\right]_{\mathrm{n}}$ can be used for GaSb thin film production whereas the precursor (1) is the most promising candidate for a MOCVD process under HV conditions. The film density and roughness can easily controlled by changing the substrates temperature during the 
deposition process at a given geometry. The achieved GaSb films were pure when clean $\mathrm{Si}(001)$ wafers were used as a substrate. Carbon and oxygen contaminations could be found at the surface only, which was due to the ambient conditions while storing the films.

\section{Acknowledgements}

This work was financially supported by the Deutsche Forschungsgemeinschaft (Schwerpunktprogramm 1119).

\section{Literature:}

[1] A. Kuczkowski, S. Schulz, M. Nieger, P. Saarenketo, Organometallics 20, 2000-2006 (2001)

[2] S. Schulz, S. Fahrenholz, A. Kuczkowski, W. Assenmacher, A. Seemayer, A. Hommes, K. Wandelt, Chemistry of Materials 17, 1982-1989 (2005)

[3] T.D. Veal, M.J. Lowe, C.F. McConville, Surface Science 499, 251-260 (2002)

[4] F.M. Liu, L.D. Zhang, Semiconductor Science and Technology 14(8), 710-714 (1999)

[5] W. Yu, J.L. Sillivan, S.O. Saied, Surface Science 352-354, $781-787$ (1996) 


\begin{tabular}{|c|c|c|c|}
\hline Peak & binding energy $[\mathrm{eV}]$ & splitting $[\mathrm{eV}]$ & ratio \\
\hline Ga3d & $18,77(20)$ & $0,446(38)$ & $0,665(93)$ \\
\hline Sb4d & $31,72(20)$ & $1,269(33)$ & $0,733(83)$ \\
\hline Ga3p & $104,02(20)$ & $3,36(15)$ & $0,425(86)$ \\
\hline Sb3d & $527,80(20)$ & $9,368(32)$ & $0,676(5)$ \\
\hline
\end{tabular}

table 1: peak position and spin orbit splitting of $\mathrm{Ga}$ and $\mathrm{Sb}$ photoelectrons

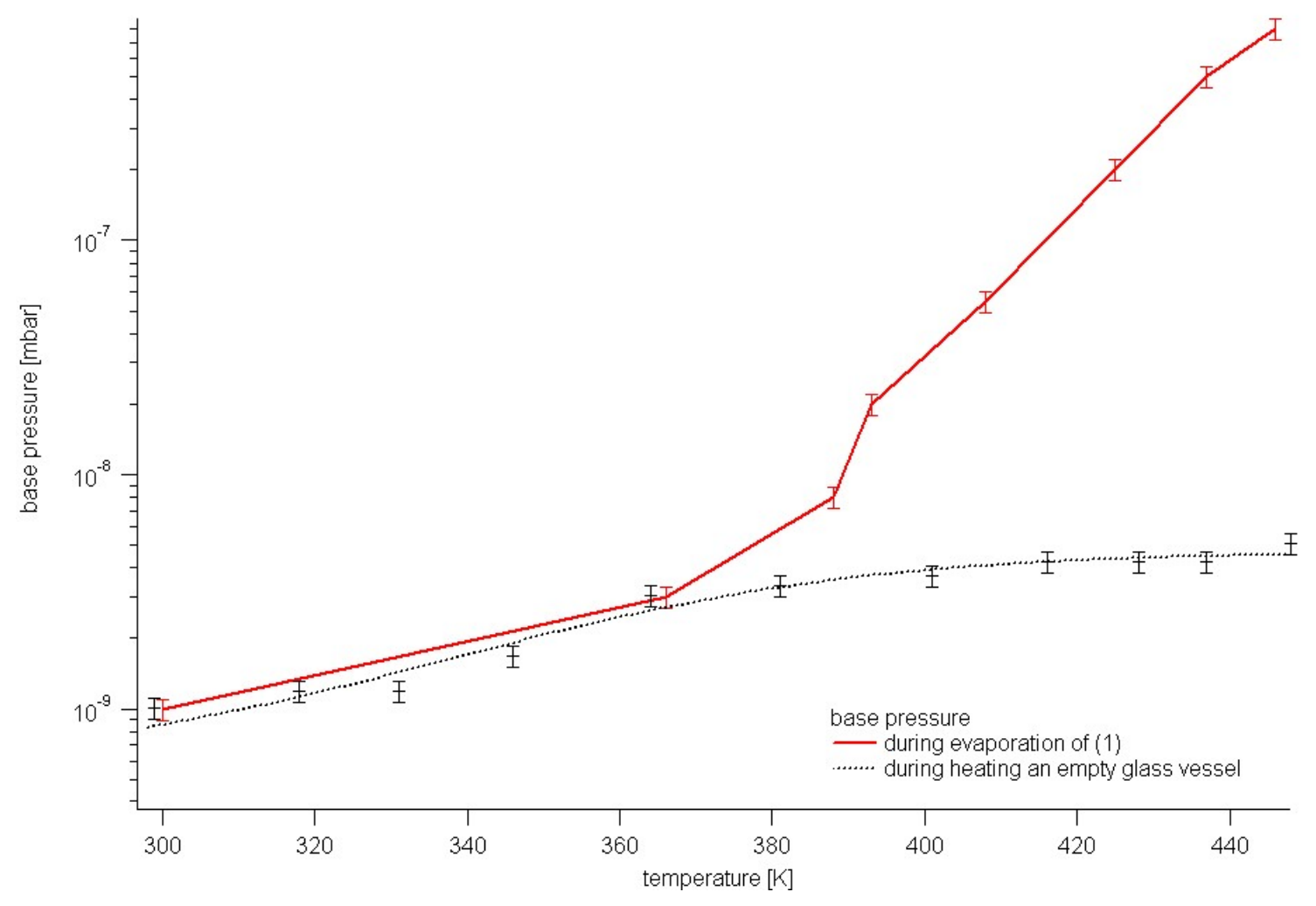

figure 1: base pressure during evaporation of (1) 

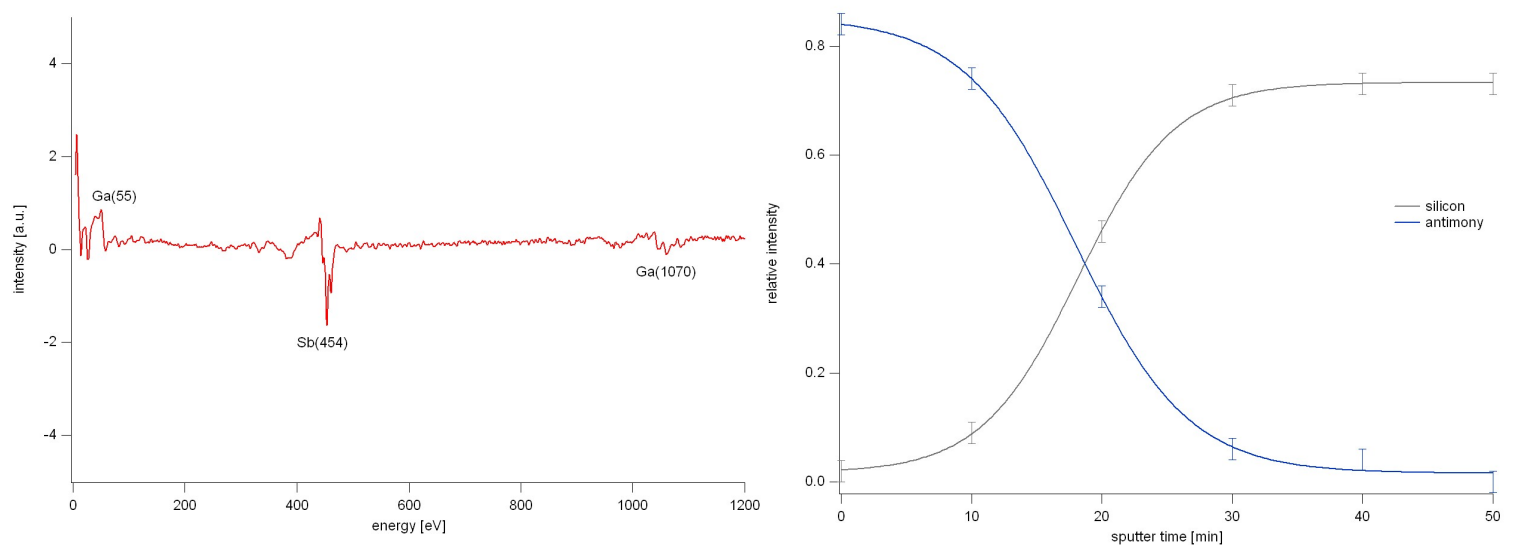

figure 2a: auger spectrum of a sputtered uhv grown film figure $2 \mathrm{~b}$ : relative intensity of silicon and antimony auger signals as a function of sputter time

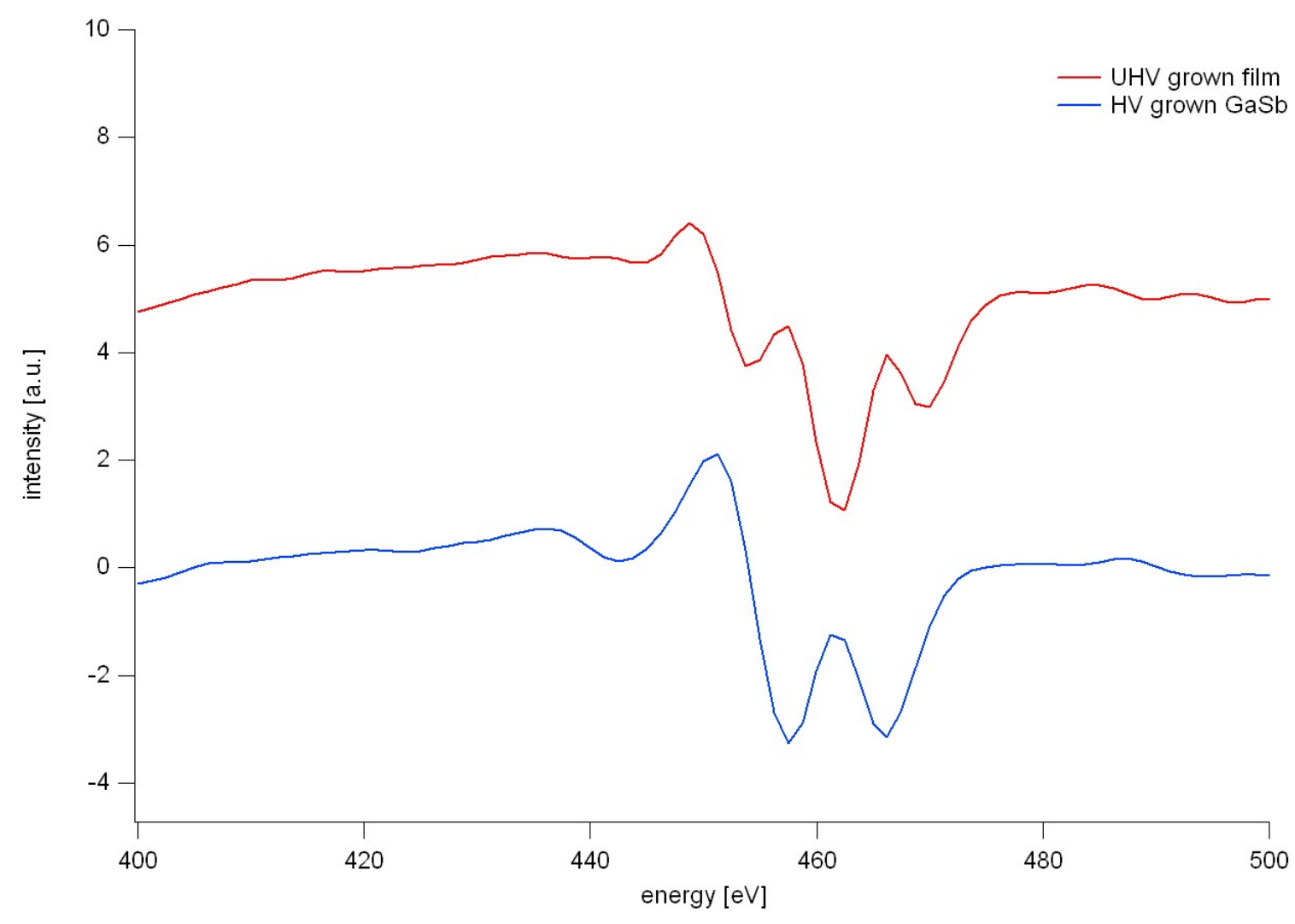

figure 3: SB MNN photoelectrons of the hv-grown and the uhv-grown film 


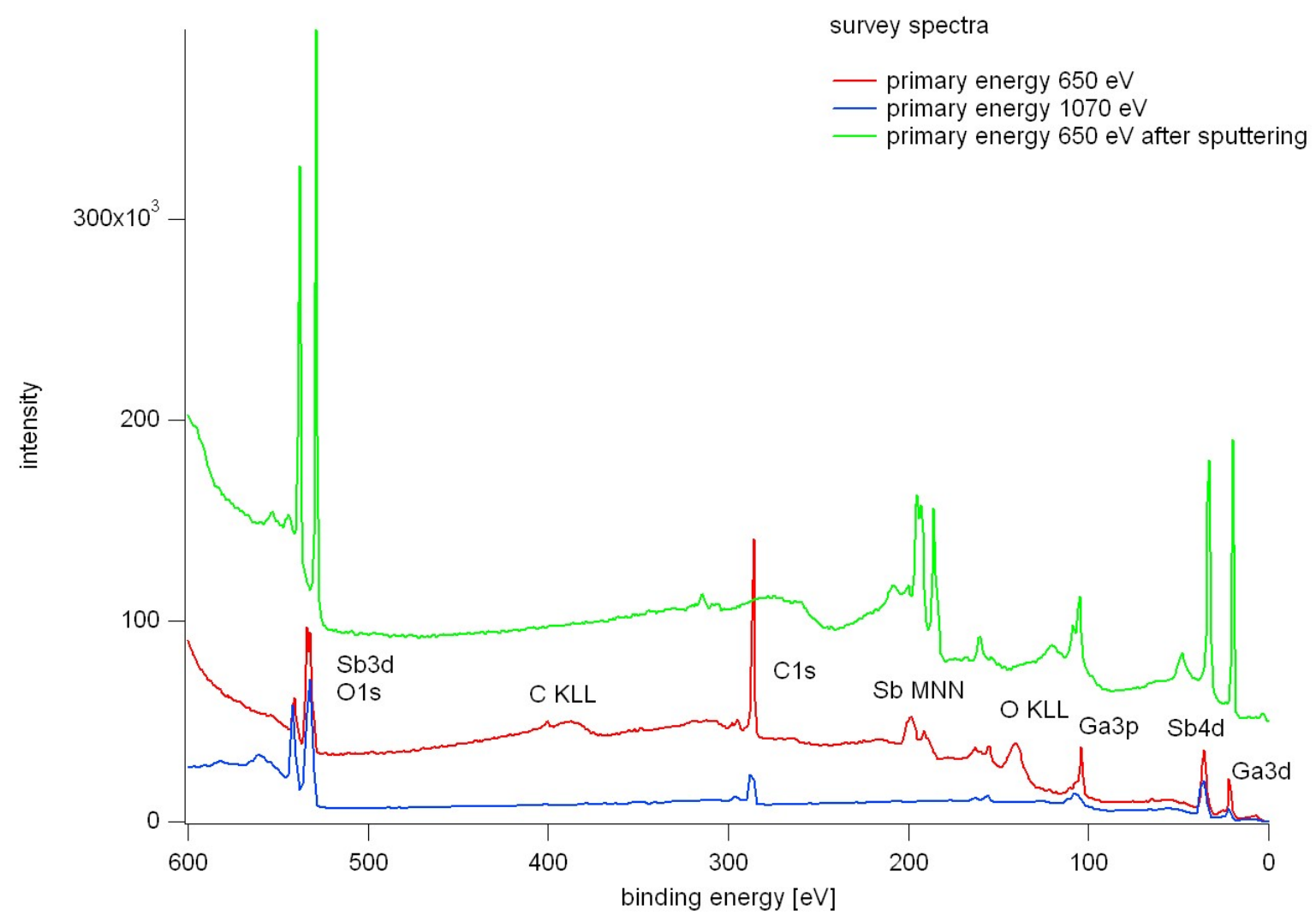

figure 4: s-xps survey spectra at different primary energies

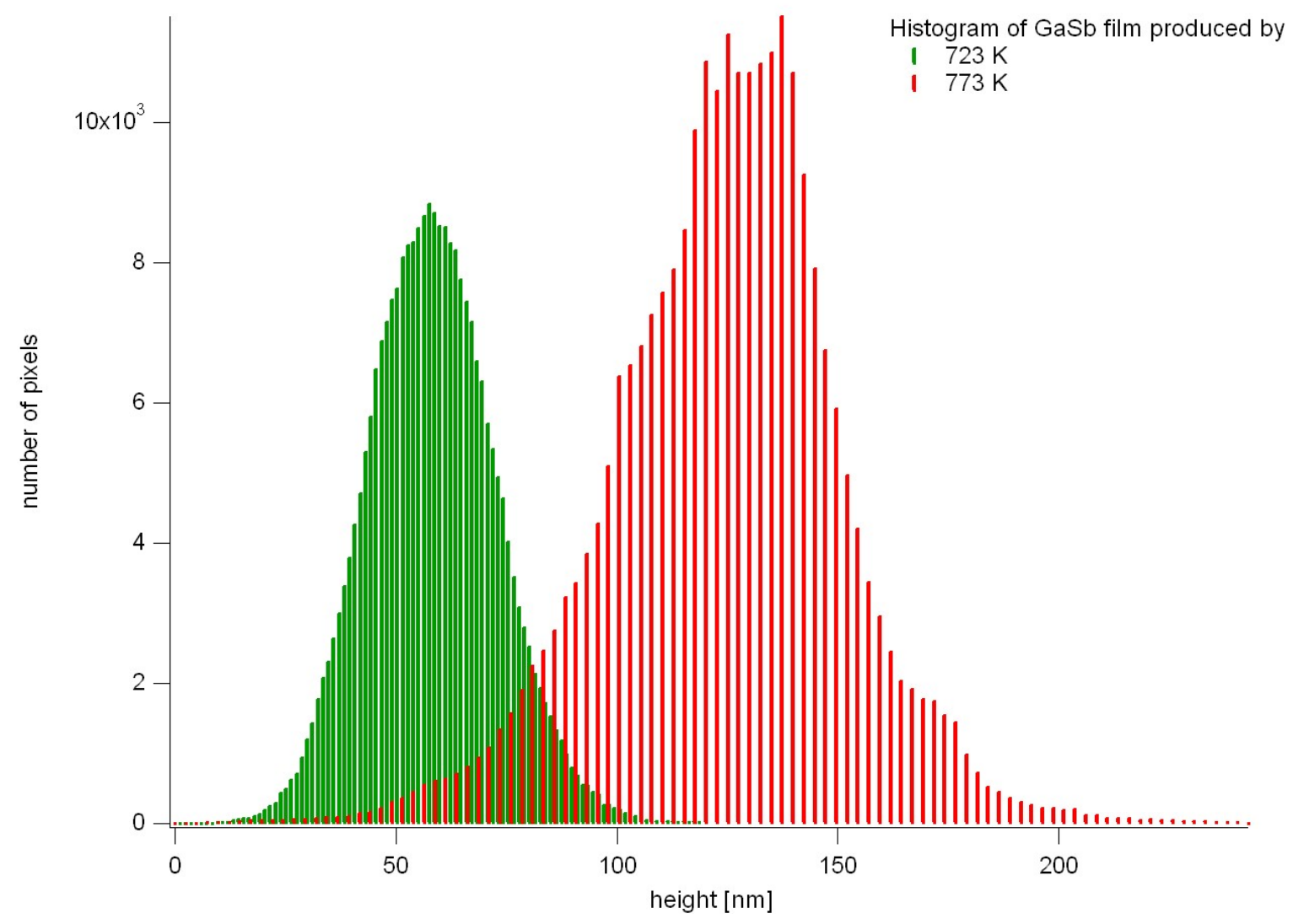

figure 5: histogram of afm topography images of films deposited at different temperatures 


\section{DuEPublico}

DEU S ISE

\section{Duisburg-Essen Publications online}

offen im Denken

Ub $\mid \begin{aligned} & \text { universitäts } \\ & \text { bibliothek }\end{aligned}$

This text is made available via DuEPublico, the institutional repository of the University of Duisburg-Essen. This version may eventually differ from another version distributed by a commercial publisher.

DOI: $\quad 10.1016 /$ j.jcrysgro.2008.07.114

URN: urn:nbn:de:hbz:464-20201027-082457-6

This is the "Authors Accepted Manuscript" of an article finally published in: Journal of Crystal Growth, Volume 310, Issue 23, 2008, Pages 4831-4834. DOI: 10.1016/j.jcrysgro.2008.07.114 